\title{
Case Series of Hemophagocytic Lymphohistiocytosis in Buali Hospital, Ardabil, Iran in July 2018
}

\section{Fathi Afshin' ${ }^{1}$, Adel Ahadi ${ }^{1}$, Maskani Reza ${ }^{1 *}$, Hosseini Anbaran Sonia ${ }^{2}$, Rashid Sheikh Ahmad Fatima ${ }^{2}$ and Ghorbani Maryam $^{1}$}

${ }^{1}$ Pediatric Department, Buali Children's Hospital, Ardabil University of Medical Sciences (ARUMS), Iran

${ }^{2}$ Department of Medical, Ardabil Branch, Ardabil University of Medical Sciences (ARUMS), Iran

*Corresponding Author: Maskani Reza, Pediatric Department, Buali Children's Hospital, Ardabil University, of Medical Sciences (ARUMS), Iran.

Received: August 07, 2019; Published: September 06, 2019

DOI: 10.31080/ASPE.2019.02.0139

\begin{abstract}
We present an one year-old boy and a 4-month old girl from Iran with hemophagocytic lymphohistiocytosis (HLH) associated with Leishmania.

HLH is not an independent disease but rather a life-threatening clinical syndrome that occurs in many underlying conditions and in all age groups [1]. It is commonly appears in infancy [2]. In our cases, treatment with Amphotericin B was started and first case responded and second case unfortunately died.
\end{abstract}

Keywords: Visceral Leishmaniasis, Hemophagocytic Lymphohistiocytosis, Hepatosplenomegaly

\section{Introduction}

Hemophagocytic lymphohistiocytosis (HLH) is a syndrome of pathologic immune activation characterized by clinical signs and symptoms of extreme inflammation [3]. HLH is a syndrome that presents in many forms: fever of unknown origin (FUO), hepatitis/acute liver failure, sepsis-like, Kawasaki-like, and neurologic abnormalities. Not all of the HLH diagnostic criteria may be present initially, so it is important to follow clinical signs and laboratory markers of pathologic inflammation repeatedly to identify the trends [3]. Some reports of hemophagocytic lymphohistiocytosis (HLH) secondary to leishmaniasis have been published, most of which referring to young children $[4,5]$.

\section{Case Presentation}

Case 1

A one-year-old boy was admitted to the Buali Hospital, Ardabil, Iran on July 2018, with a 3-week history of fever, Hepatosplenomegaly and pancytopenia. Serum testing showed elevated transaminase levels (ALT: 750, AST: 820), hypertriglyceridemia (TG:420), hyperferritinemia (Ferritin:9780) and fibrinogen level was less than 50 unit. Hemophagocytic lymphohistiocytosis was diagnosed on bone marrow examination that showed HLH with Leishmania body (Figure 1). The patient was tested for various infectious agent that all of them were negative expect Leishmania, (HBS Ag: negative HCV Ab: negative HIV Ab: negative), DAT for Leishmania was positive. Treatment with amphotericin B results in a dramatic resolution of all signs and symptoms within 10 day.

Case 2

4 month old girl was admitted to the hospital because of fever, pallor and Hepatosplenomegaly for 2 week. Intermittent high grade fever persisted with anorexia and weight loss. Examination revealed fever, pallor and petechiae. Abdominal examination revealed Hepatosplenomegaly. Respiratory, cardiac and neurological were normal. Lab data showed pancytopenia, liver function tests were abnormal (ALT: 672 AST: 1449 ALP: 386 Bill Total: 8 Bill Di- 
rect: 3.6). Ferritin was very high (16493). Coagulogram revealed coagulopathy and hyperfibrinogenemia. Renal function and electrolytes were normal. Abdominal sonography showed H€ splenomegaly. DAT WAS 1/1600. Fever persisted and amphot $\mathrm{B}$ was started. Bone marrow examination (BMA) revealed hemophagocytosis with leishman bodies. (Figure 1). After one wet doesn't have response to antibiotic. Dexamethasone and Eupuside were added for HLH. But unfortunately she didn't response to treatments and died.

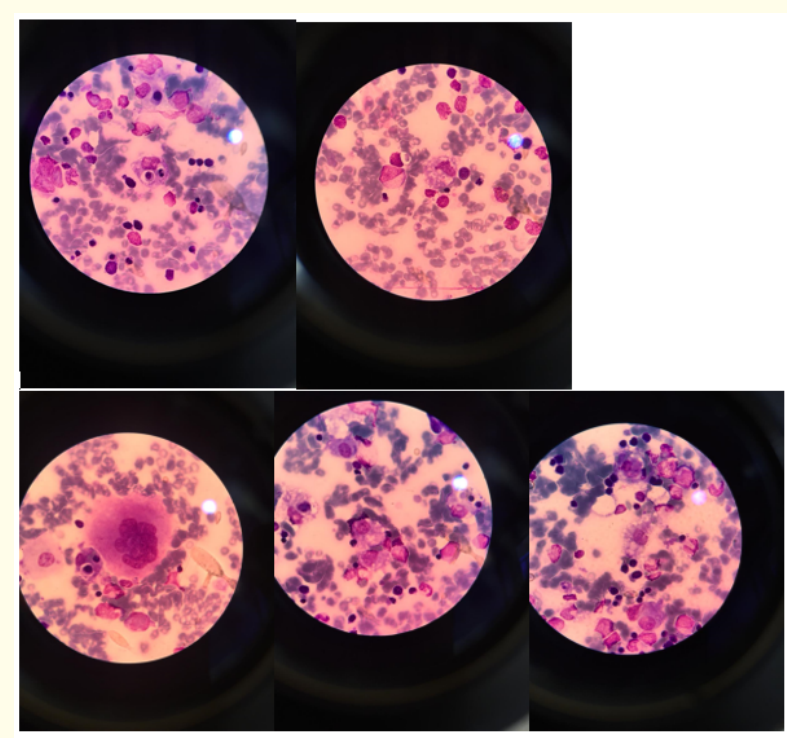

Figure 1

\section{Conclusion}

In conclusion, HLH secondary to Leishmania is extremely rare and potentially fatal. The diagnosis is often missed due to overlapping clinical features and negative bone marrow. A high index of clinical suspicion, repeated marrow evaluation with culture and or serology is often required to establish leishmaniasis [6]. Liposomal amphotericin is the drug of choice. Leishmania must be considered and excluded in patients with HLH before immunosuppression is considered.

\section{Bibliography}

1. Janka GE and Lehmberg K. "Hemophagocytic lymphohistiocytosis: pathogenesis and treatment". Hematology-American Society of Hematology Education Program (2013): 605-611.

2. Schwartz RA. "Lymphohistiocytosis (Hemophagocytic Lymphohistiocytosis)". Medscape (2016).

3. Jordan MB., et al. "How I treat hemophagocytic lymphohistiocytosis”. Blood 118.15 (2011): 4041-4052.

4. Rajagopala S., et al. "Visceral leishmaniasis associated hemophagocytic lymphohistiocytosis-case report and systematic review". Journal of Infection 56 (2008): 3810-388.

5. Tapisiz A., et al. "Hemophagocytic lymphohistiocytosis associated with visceral leishmaniasis". Journal of Tropical Pediatrics 53.5 (2007): 359-361.

6. Agarwal S., et al. "Hemophagocytic syndrome associated with leishmaniasis". Indian Journal of Pediatrics (2006): 73-446.

\section{Volume 2 Issue 10 October 2019}

(C) All rights are reserved by Maskani Reza., et al. 